The use of a high-order MEMS deformable mirror in the Gemini Planet Imager

L. A. Poyneer, B. Bauman, S. Cornelissen, S. Jones, B. Macintosh, D. Palmer, J. Isaacs

January 5, 2011

MEMS Adaptive Optics V

San Frnacisco, CA, United States

January 27, 2011 through January 27, 2011 
This document was prepared as an account of work sponsored by an agency of the United States government. Neither the United States government nor Lawrence Livermore National Security, LLC, nor any of their employees makes any warranty, expressed or implied, or assumes any legal liability or responsibility for the accuracy, completeness, or usefulness of any information, apparatus, product, or process disclosed, or represents that its use would not infringe privately owned rights. Reference herein to any specific commercial product, process, or service by trade name, trademark, manufacturer, or otherwise does not necessarily constitute or imply its endorsement, recommendation, or favoring by the United States government or Lawrence Livermore National Security, LLC. The views and opinions of authors expressed herein do not necessarily state or reflect those of the United States government or Lawrence Livermore National Security, LLC, and shall not be used for advertising or product endorsement purposes. 


\title{
The use of a high-order MEMS deformable mirror in the Gemini Planet Imager
}

\author{
Lisa A. Poyneer ${ }^{a}$, Brian Bauman ${ }^{a}$, Steven Cornelissen ${ }^{b}$, Joshua Isaacs $^{a, c}$, Steven Jones ${ }^{a}$, Bruce \\ A. Macintosh ${ }^{a}$, and David W. Palmer ${ }^{a}$ \\ ${ }^{a}$ Lawrence Livermore National Laboratory, 7000 East Ave, Livermore, CA, 94550 \\ ${ }^{b}$ Boston Micromachines Corporation, 30 Spinelli Place, Cambridge, MA 02138 \\ ${ }^{c}$ now at University of Wisconsin-Madison, 1150 University Avenue, Madison, WI 53706
}

\begin{abstract}
We briefly review the development history of the Gemini Planet Imager's 4K Boston Micromachines MEMS deformable mirror. We discuss essential calibration steps and algorithms to control the MEMS with nanometer precision, including voltage-phase calibration and influence function characterization. We discuss the integration of the MEMS into GPI's Adaptive Optics system at Lawrence Livermore and present experimental results of 1.5 $\mathrm{kHz}$ closed-loop control. We detail mitigation strategies in the coronagraph to reduce the impact of abnormal actuators on final image contrast.
\end{abstract}

Keywords: Adaptive Optics, MEMS deformable mirror, Gemini Planet Imager, wavefront control

\section{INTRODUCTION}

The Gemini Planet Imager (GPI) ${ }^{1}$ is a hyperspectral coronagraph designed to directly image extra-solar planets. It uses high-performance adaptive optics $(\mathrm{AO})$ to reduce dynamic residual wavefront error to a few tens of $\mathrm{nm}$ RMS. A spatially-filtered wavefront sensor ${ }^{2}$ and a deformable mirror (DM) with a few thousands of actuators are essential to achieving low residual wavefront error in the presence of atmospheric turbulence. GPI's science-driven design specifies 18-cm subapertures. Given the $7.77 \mathrm{~m}$ Gemini South pupil, this requires at 43 subapertures and hence 44 actuators across the circular pupil.

There are two major technology options for such a high-order deformable mirror: piezoelectric-actuator mirrors (such as those produced by Cilas and Xinetics) and MEMS mirrors. The GPI instrument design is highly constrained by operating at Cassegrain focus on the back side of the Gemini primary mirror, unlike many AO systems (such as SPHERE) which are at Nasmyth. ${ }^{3}$ The Cassegrain environment limits the overall size, mass and power of GPI, making a compact, low-power MEMS DM very attractive.

At the time of the original conceptual design by our team for adaptive optics planet imager (see Macintosh et $\mathrm{al}^{4}$ ), a MEMS with enough actuators and adequate stroke did not exist. In 2004 a multi-phase development process was initiated, funded by the NSF Center for Adaptive Optics, the UCSC Moore Laboratory for Adaptive Optics (LAO), and the Gemini Observatory.

\section{DEVELOPMENT OF MEMS DM}

Initial specifications for the 4,096-actuator $(64 \times 64$, more easily referred to as " $4 \mathrm{~K}$ ") MEMS DM for GPI (hereafter referred to as the "GPI MEMS") were drafted in 2004. It took nearly two years of study and testing to finalize the requirements.

Beginning with Phase 1, BMC developed new actuator designs to increase device stroke significantly and to improve the surface quality of the mirrors. In Phase 2, three candidate actuator designs were selected; 1,024 actuator $(32 \times 32$, more easily referred to as " $1 \mathrm{~K}$ ") mirrors of each design were then fabricated. These mirrors were tested at BMC and also extensively at the LAO. ${ }^{5-10}$ The final, winning design was based on the actuator used in the BMC 140 MEMS. This actuator has a total stroke of more than 4 microns (for the center of a $3 \times 3$

Send correspondence to Lisa Poyneer: poyneer1@llnl.gov, 19254233360 
actuator patch) along with excellent surface quality of less than $5 \mathrm{~nm}$ RMS surface print-through and actuator scalloping.

Early in our testing of $1 \mathrm{~K}$ devices, humidity was identified as a significant threat to device function. At high levels of humidity and voltage, oxidation of the exposed electrodes occurs, leading to permanent actuator failure. During 4K GPI MEMS development, the wiring layout was changed to sandwich the traces between layers of dielectric during manufacturing in order to accommodate all the necessary wires. This had the side effect of significantly reducing the amount of exposed wiring and hence resulted in more graceful failure in the presence of humidity; actuator stroke is gradually reduced as oxidation occurs.Despite this design change, humidity is still a concern for GPI. The final instrument has the MEMS in a sealed enclosure, and that enclosure is constantly supplied with dry clean air at a slight overpressure.

During Phase 2, device yield was significantly improved. This means fewer actuators had faults created during manufacturing. This improvement was obtained by trouble-shooting the manufacturing process. For example, many electronic shorts were caused by small pinholes in the films during manufacture.

Phase 3 of the GPI MEMS development entailed fabrication of the final $4 \mathrm{~K}$ device. Testing at the LAO continued. ${ }^{11}$ The final phase required two foundry runs to produce a device with a sufficiently low number of bad actuators. Despite the improvements made in device design and manufacturing of $1 \mathrm{~K}$ mirrors during Phase 2 , the fabrication of the larger $4 \mathrm{~K}$ mirrors in Phase 3 posed a unique challenge. It was always recognized that the process was unlikely to result in a $4 \mathrm{~K}$ device with no defective actuators. An early mitigation strategy for device defects was to exploit the fact that the active GPI pupil is 43 actuators in diameter. This means that a $4 \mathrm{~K}$ device could have bad actuators, but as long as a full 43-actuator-across pupil could be found with no bad actuators, the device would be usable. Based on the defect rates observed in $1 \mathrm{~K}$ devices, we believed this was practical.

However, the average number of defective actuators in a $4 \mathrm{k}$ device turned out to be not simply four times that of a $1 \mathrm{k}$ device. The larger number of actuators leads not only to a larger mirror surface area, but to a much larger wiring area. This means fewer devices per $15-\mathrm{cm}$ wafer and fewer chances to get a device with no defects from a single foundry run; due to the higher density, a single foundry run could produce one thousand candidate $1 \mathrm{~K}$ devices, but only fifty $4 \mathrm{~K}$ devices. ${ }^{12}$

In the end no 4K device met this. The top candidate 4K GPI MEMS has eight stuck or nearly-dead actuators. In the best GPI geometry, one will be hidden behind the secondary obscuration; the other two are just inside the outside edge of the circular pupil. In addition, six pairs of actuators are weakly electrically coupled to each other, but the closed-loop control can account for this with a slight loss of net stroke.

In Section 3 we discuss in detail the methods developed to provide nm-level control of the surface of the MEMS DM. We then present in Section 4 experimental results from integration of the engineering-grade 4K GPI MEMS with the GPI real-time computer and hardware. Section 5 describes the optical modifications to the GPI coronagraph that will ameliorate the effects of the remaining bad actuators. Finally, we wrap up with the path forward to GPI deployment at Gemini South in 2011.

\section{TECHNICAL CHALLENGES IN USING A MEMS DM}

\subsection{Woofer-Tweeter control}

Despite the large increase in stroke that occurred during the GPI MEMS development process, a single MEMS DM does not have adequate stroke to fully correct typical atmospheric turbulence. Under Kolmogorov theory, low spatial frequency phase errors have the largest amplitude. A low-order, high-stroke DM can be used as a Woofer to correct the low spatial frequencies, leaving the higher frequency, lower amplitude errors for the MEMS. Lavingne and Véran ${ }^{13}$ provide a detailed discussion of the GPI Woofer parameter specification process and the control algorithms necessary to split the phase between the two mirrors. 


\subsection{Influence function superposition}

The response of the surface of a DM to a single poked actuator is termed the "influence function". A common way to characterize the influence function is to determine the coupling at the nearest neighbor actuator, ${ }^{14}$ as the ratio of the peak height to the neighbor's height. An ideal DM would have an influence function which is a sinc function. A sinc influence function has zero coupling at all other actuators. Realistic influence functions can be modeled as Gaussians or sums of Gaussians. In that case, the larger the nearest-neighbor coupling, the broader the influence function.

If a MEMS DM was a pure linear, shift-invariant (LSI) device, this influence function (and its Fourier domain pair, the transfer function) would be all that is necessary to completely characterize the surface shape. A simple precompensation for the influence function, accomplished as a division in the Fourier domain by the magnitude of the transfer function, would allow accurate control of the mirror surface.

A MEMS mirror, in which the actuator force for a given voltage is a nonlinear function of the current actuator position, is not a LSI system. This can be trivially calibrated for an isolated actuator, but not for the whole ensemble of actuators. At least three different efforts have worked towards detailed modeling of the face sheet motion and how to control that surface with high precision. ${ }^{15-19}$ However, our own work has shown that the non-linearity due to the face sheet is low enough that GPI does not require an advanced algorithm to determine actuator voltages. ${ }^{20}$ Calibration of the quadratic voltage-phase relationship (see below) and the influence function are all that is necessary.

The requirement for the GPI MEMS is that the nearest-neighbor coupling be less than $35 \%$. This broad influence function translates into a narrow frequency response that attenuates high spatial frequencies. Given the finite voltage range, this means that the mirror can make less peak to valley phase at a high spatial frequency than at a low. Morzinski et $\mathrm{al}^{10}$ have shown that for the Phase $21 \mathrm{~K}$ mirrors, the highest frequency sinusoids can only be made with one-third the stroke of the lowest spatial frequency sinusoids. For that test the sinusoids were made with same peak-to-valley voltage range regardless of spatial frequency.

\subsection{Voltage-phase calibration}

Unlike piezoelectric actuators, where the stroke is a linear function of the applied voltage, ${ }^{14}$ MEMS actuators have stroke proportional to the square of the applied voltage. As such, there needs to be an explicit estimationfitting process. First the phase correction that is desired on the surface of the MEMS is estimated. Second, this phase is fit by inverting the quadratic relationship to determine the appropriate voltage for each actuator.

This inversion process requires precise knowledge of the response of each actuator. This can be measured with an interferometer, as was done in our testing at the LAO (see, for example, Figs 3 and 5 of Evans et $\mathrm{al}^{8}$ ). Multiple devices have been measured during the MEMS development phases. The quadratic voltage-phase response is relatively uniform across the device for normal actuators.

We have further determined that the best measurement to use in determining the calibration curve is a spatially-filtered phase measurement. ${ }^{20}$ For the lower-stroke $1 \mathrm{~K}$ devices, this was easily obtained by low-pass filtering the high resolution interferometric phase estimate down to the Nyquist limit imposed by the actuator spacing. For the GPI MEMS with higher stroke, neither our PSDI nor GPI's own spatially-filtered ShackHartmann ${ }^{2}$ have adequate dynamic range to measure a full actuator poke. We will instead rely on a calibration done with a Zygo interferometer.

\subsection{Abnormal actuators}

Throughout the GPI MEMS development process, several types of abnormal actuators have been identified and studied. For example, see section 3.2 of Evans et al. ${ }^{8}$ During our most recent development, we have worked with both $1 \mathrm{~K}$ and $4 \mathrm{~K}$ DMs that have several types of abnormal actuators.

Instead of considering a taxonomy of the many types of abnormal actuators, we now approach the issue from the perspective of how well we can sense and control them.

We begin by considering how to control (or fit the phase) to abnormal actuators. For normal actuators, as described above, we do influence function pre-compensation and then invert the measured quadratic voltagephase calibration. Infrequently the desired phase on an actuator is out of the commanded range. In this case, to 
prevent integrator windup, we truncate the voltage if it outside the valid range and then clip, by sending back this truncation error signal to the integrator. At each time step this updates the integrator for that actuator to the phase that was actually commanded, not the phase that was desired. This prevents windup and ensures prompt resumption of excellent wavefront correction when the saturation event is over. Throughout this process, each actuator is handled independently, as the influence function precompensation takes care of LSI coupling.

All abnormal actuators must also be controlled in this framework, with small, computationally efficient variations in control being permissible. Some abnormal actuators will require no modification beyond a custom voltage-phase curve. A "weak" actuator, which is characterized as not having the full stroke range, will simply have its own voltage-phase curve that reflects this. It will saturate much more frequently, but clipping will ensure no windup. Other actuators will simply not respond at all. These effectively have a voltage-phase curve where the minimum and maximum voltage are the same. This actuator will effectively always be saturated and is permanently clipped to bias voltage (zero phase). This method of custom voltage-phase calibrations and custom clipping was implemented in our IDL-based, 1K MEMS testbed in 2009 and proved effective.

When actuators are coupled to each other we no longer can fit them independently. The two final candidate GPI MEMS devices each have several coupled sets of actuators. As long as the behavior is deterministic, we should be able to characterize it and implement a fitting and integrator-update that makes the phase that we want. Given the desired phases at the coupled actuators, our strategy is to determine the best reachable phase for the actuators, and the corresponding voltages. The integrator values must then be updated to reflect the commanded phases, not the estimate ones, using a procedure similar to clipping.

One important aspect of the device characterization is the determination of the reachable space of phases for two actuators that are coupled to each other. For some coupled pairs it is already clear that certain phase combinations are not possible (e.g. one actuator at maximum phase, the other at minimum). In that case, the fitting will have to be based on some reasonable error criterion, such as minimizing the squared error from the desired phases.

This remains an outstanding research and implementation item on the final GPI MEMS. We assume that the number of abnormal actuators is very small. Since we are limited by computational and programming work-time constraints, we will not take a unified, optimal control approach to the problem.

The second aspect of dealing with abnormal actuators is their effect on the WFS spots. Abnormal actuators will not be able to shape the estimated phase some or all of the time. This will result in small influence-function sized regions of phase error, potentially with amplitude of hundreds of $\mathrm{nm}$ to a few microns. These abnormal actuators present a significant challenge to final science contrast, as is described in Section 5. They also directly effect the wavefront sensor. The first effect is obvious - the mis-positioned actuator leaves a large phase gradient, which causes large phase slopes in the four subapertures which surround that actuator (in our Shack-Hartmann sensor).

For some abnormal actuators this information is useless - if we can't control the actuator, there's no need to know that it is mis-positioned. If we zero-out the $\mathrm{x}$ - and $\mathrm{y}$-slopes in the four surrounding subapertures, this has the effect of forcing the phase to be as flat as possible on that actuator relative to its neighbors. This is a sensible position for the actuator. Another reason blocking these large phase excursions is sensible is due to the Fourier-domain nature of our control. A pure impulse (a signal 1 at that actuator and zero at all others) has a Fourier transform with uniform power at all spatial frequencies. This means that sensing a mis-positioned actuator results in a phase with a (potentially high amplitude) impulse. This impulse contributes power to the low spatial frequencies that are handed off to the Woofer, as well as to the high spatial frequencies that will be amplified by the influence function pre-compensation filter. Removing the impulse, especially if that actuator is uncontrollable, will prevent contamination of the Fourier modes and should result in better performance.

A further reason to zero out the slopes is that we are using a spatially-filtered Shack-Hartmann. A unique effect with this sensor is that a large phase excursion on a single actuator causes a significant reduction in spot intensity in the four neighboring subapertures. Figure 1 shows results of a Fourier optics simulation of our sensor, giving the intensity level in a directly adjacent subaperture due to a phase excursion on that actuator. For a regular aliased Shack-Hartmann, little change is seen out to nearly a wave and a half. In contrast, the mis-positioned actuator scatters light outside the $\lambda /(2 d)$ radius of the spatial filter, which leads to a drop-off in 


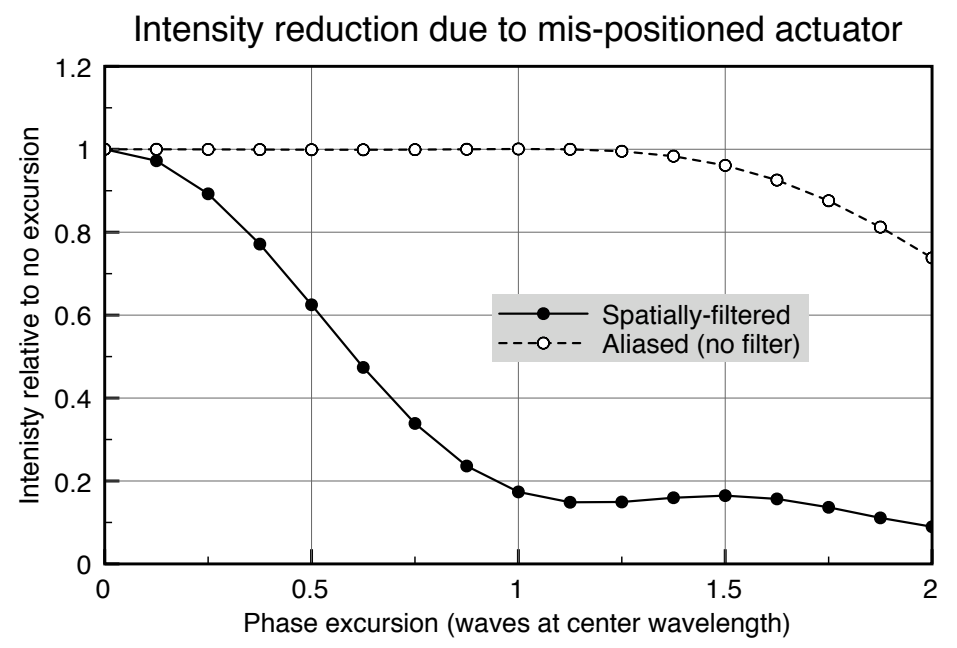

Figure 1. When an actuator is mis-positioned and causes a phase bump away from flat with its neighbors, the spatial filter causes a significant reduction in light in the immediately neighboring subapertures. Results from a Fourier Optics simulation of the GPI WFS.

intensity. The effect is noticeable for only a quarter of a wave. By a full wave (800 $\mathrm{nm}$ for GPI), the intensity is just $17 \%$ of normal. This loss of intensity means much more noise in that subaperture. For large excursions this is equivalent to reducing the guide-star brightness by nearly two full magnitudes in those subapertures. Preventing extremely noisy measurement is a second good reason to zero-out the slopes before reconstruction.

During our use of the engineering-grade GPI MEMS at LLNL in 2010, we had on order 1 dozen abnormal actuators in the pupil. As we did not have at that time the clipping and cleanup code implemented in the RTC, we chose to block the slopes surrounding all misbehaving actuators to prevent integrator windup. This proved sufficient for good performance and was probably not a limiting factor; see Section 4 for discussion of our experimental results.

With the final device a more rigorous evaluation of which actuators should always have their surrounding slopes zeroed-out, and which can be sensed will be conducted.

\subsection{Optical design}

The small form factor of the GPI MEMS, which is a boon for building a space-constrained instrument, presents unique challenges for instrument optical design. With 400 micron spacing of the actuators, the GPI pupil at the MEMS is $1.72 \mathrm{~cm}$. Given the $7.77 \mathrm{~m}$ primary of Gemini South, this means that the pupil size is reduced by a factor of 452. Correspondingly, the field angles at the telescope pupil are increased by the same factor at the MEMS. Each arcsecond of field of view at the telescope translates to 452 arcseconds, or an eighth of a degree at the MEMS. For a typical general-purpose AO system (but with a MEMS), a field of an arcminute would require an optical design that is well-corrected for a nearly 8-degree field. That is very challenging. However GPI has a narrow field of view of 2.8 arcseconds. This is about a third of a degree at the MEMS, obviating this optical design challenge.

A second unique issue is diffraction effects. We know that as beams propagate, they evolve in amplitude and phase so that a phase aberration generated at an optical surface turns into some other phase and amplitude pattern by the time we measure the wavefront at the pupil of the system. To some extent, the phase generated at an optical surface turns into an amplitude at the pupil of the system, and amplitude variations cannot be corrected in a single-conjugate system. In general, as the beam sizes become smaller, these propagation effects increase. A convenient concept for gauging diffraction effects is Talbot imaging. In Talbot imaging, we start with a wavefront of sinusoidal phase and uniform amplitude. After a certain distance, the beam has evolved into one of uniform phase and sinusoidal amplitude. The beam continues evolving until it finally returns to its 
original sinusoidal phase and uniform amplitude. The distance over which the full cycle occurs is called the Talbot length, and it is a function of the spatial period of the sinusoid: $2 a^{2} / \lambda$, where $\lambda$ is the wavelength in meters and $a$ is the spatial period in meters. ${ }^{21}$

For large spatial periods, the Talbot length is long and unnoticeable. But for GPI, we are concerned with spatial periods on the order of 2 actuator pitches (800 microns) with a corresponding Talbot length of $\sim 1 \mathrm{~m}$ (for both the WFS and science light). For this scale, a sinusoidal phase turns into an amplitude in $250 \mathrm{~mm}$, which is approximately the same as the distance between the MEMS and its neighboring optics. The immediate conclusion is that surface figure with short periods will be (mostly) uncorrected in the system, and thus require tight tolerances. To control these effects, the mirrors in the GPI system were manufactured to very precise optical requirements; a typical GPI mirror has a RMS wavefront error of $0.5 \mathrm{~nm}$ at mid spatial frequencies (4-22 cycles per beam). Longer spatial periods are more acceptable because they evolve much more slowly. For example, if a neighboring mirror has a sinusoidal phase with a period of $8 \mathrm{~mm}$, the Talbot length is $\sim 100 \mathrm{~m}$, and the beams remains almost unchanged after a distance of $250 \mathrm{~mm}$. The DM can then correct this phase aberration.

In the WFS, these diffraction effects are even more pronounced because the beam is very small: $\sim 2.5 \mathrm{~mm}$. The highest spatial frequency handled in GPI has a period of $a=125$ microns in this reduced beam, so the Talbot length is only $40 \mathrm{~mm}$. In other words, if the lenslet array is misplaced axially by only $10 \mathrm{~mm}$, then we will not measure the highest spatial frequency at all. In addition, Talbot effects show up on the other side of the lenslets: the lenslets create a periodic set of (intensity) dots at a period of 63 microns, for a Talbot length of $10 \mathrm{~mm}$ at the WFS center wavelength. This dot amplitude pattern itself evolves into phase (the dots actually disappear) and back into dots again $5 \mathrm{~mm}$ later. In our initial alignment of the WFS relay, we reimaged the wrong spot grid. This became obvious as we used the sensor because the spots, while the right size, moved far too much for a known wavefront tilt. A realignment processes using the nulls allowed us to image the correct spot image. If we had been using more commonly sized lenslets (e.g. 250 microns) this problem would not occur as the Talbot length would be large compared to the propagation distances involved.

\section{EXPERIMENTAL RESULTS: GPI AO SUBSYSTEM}

\subsection{System alignment and calibration}

In our August 2010 tests, we used an engineering-grade 4K MEMS. This MEMS had approximately a dozen abnormal actuators in the active pupil that required masking out in the WFS and were uncontrolled. The Woofer-Tweeter was implemented with a split matrix measured in-situ. Twenty-two sine and cosine pairs of the lowest spatial frequencies were sent to the Woofer. A generic quadratic voltage-phase calibration based on device usage at the LAO was used. The influence function pre-compensation filter was based on earlier high-resolution interferometric measurements of the mirror. ${ }^{22}$ To help suppress noise and non-linearities in the system (due to the engineering-grade WFS CCD), the maximum compensation for high-order modes was set to four times pre-amplification.

In addition to our previously documented precision alignment procedures ${ }^{22}$ we also used a new method for aligning the spatial filter. We exploit the reduction in intensity when an actuator is "poked" (see above) to precisely align the spatial filter in the WFS relay. Fourier Optics simulations of the WFS show that if the filter is translated off the optical axis in either $\mathrm{x}$ or $\mathrm{y}$, the intensity level in the four surrounding subapertures is proportional to that shift, along the same axis. By calculating the total intensity per subimage, we get a $2 \times 2$ signal that we can centroid on (just like a quadcell spot measurement) to determine the displacement of the spatial filter.

\subsection{Experimental methods and data analysis techniques}

Lacking a science camera with a coronagraph, we measure closed-loop performance of the AO system using its own telemetry data. The RTC can save residual phase Fourier coefficients and tip-tilt residuals at full frame rate (and many more signals)

For our closed loop testing we used a spinning phase plate. The phase plate was designed to simulate median seeing $\left(r_{0}=14.4 \mathrm{~cm}\right)$. It was rotated at an equivalent to $15 \mathrm{~m} / \mathrm{s}$, which is a higher than median wind-speed. 
The combination of our SLD light source and engineering-grade WFS CCD limited us to very high WFS SNRs. These tests were conducted at a SNR equivalent to what GPI would see for a $I=3.3$ mag star.

Our test procedure was as follows.

1. spin phase plates

2. start dumping telemetry to disk; this will later be used to construct arbitrary-length buffers

3. wait 20 seconds

4. close TT, Woofer and Tweeter loops

5. wait 20 seconds

6. turn on gain optimization

7. wait 20 seconds

8. end run and stop data collection

After the spinning plate test was completed, for calibration purposes a second test with a static plate was done. The static test followed the above procedure, expect no gain optimization was used. This provides a check on the noise levels.

Stored telemetry includes residual phases (wavefronts, microns) and residual TT (mas), as well as loop status, time stamps and other system operation information. System performance can be assessed from the residual wavefronts directly, and via a power-spectral analysis that allows estimation of performance under different conditions (e.g. modal gains).

Calculating measured error from the spatial domain signals is straight-forward. Using residual phase measurements over the 20 second interval, calculate and remove average phase (static term). For dynamic phase, calculate RMS (excluding bad actuators) across pupil for each frame. Then find the average of the interval via RSS (i.e. the square root of the average variance). For the spinning plate case, this number is the joint closed-loop measurement of temporal $\left(\sigma_{t}^{2}\right)$ and noise power $\left(\sigma_{n}^{2}\right)$. For the static plate case, this number is the single closed-loop measurement of the noise power. Because the WFS transfer function is very close to unity for temporal frequencies less than approximately $10 \%$ of the frame rate, and the phase plate is dominated by low temporal frequency error, the measured temporal power is therefore assumed to be equivalent to the error power (in the science leg). However, for the noise-only case the measured power is not the same as the error power, so an adjustment is made based on the Laplace model, assuming temporally white noise. This converts measured noise power to error noise power, which is given in the table. Temporal error is then determined via RSS (with subtraction, not addition) from the joint error and the single noise error.

Our power-spectral analysis protocol emulates the spatio-temporal Fourier analysis of the measured residual wavefront, as is done by Optimized-gain Fourier Control (OFC). ${ }^{23}$ For each frame, the Fourier coefficients are calculated from the residual phase (telemetry actually saves Fourier modes to disk, not spatial phase, so we are really just inverting an earlier processing step done for our convenience). Then for each Fourier mode from $\mathrm{k}, \mathrm{l}$ each from -23 to 24 , the temporal power spectral density (PSD) of that mode is estimated using the averaged, modified periodogram method. In the post-processing step, we can remove the mean value over the entire interval before calculating the periodogram. For 20 second intervals we have roughly 20,000 frames at $1 \mathrm{khz}$ and 30,000 frames and $1.5 \mathrm{kHz}$. The periodogram length is 2048 , giving many averages over the interval to reduce noise in the estimate.

This process produces the estimate of the joint closed-loop measurement of the dynamic atmosphere (i.e. spinning phase plate) and the WFS noise. An example of this PSD estimation is given in Figure 2. In this we see the joint PSDs closed-loop interval (black). The open-loop data provides a check on this - the red curve is the open-loop joint measurement PSD converted to estimated closed-loop measurement PSD by application of the error transfer function. There two curves agree very well, which means that our real-time controller is 

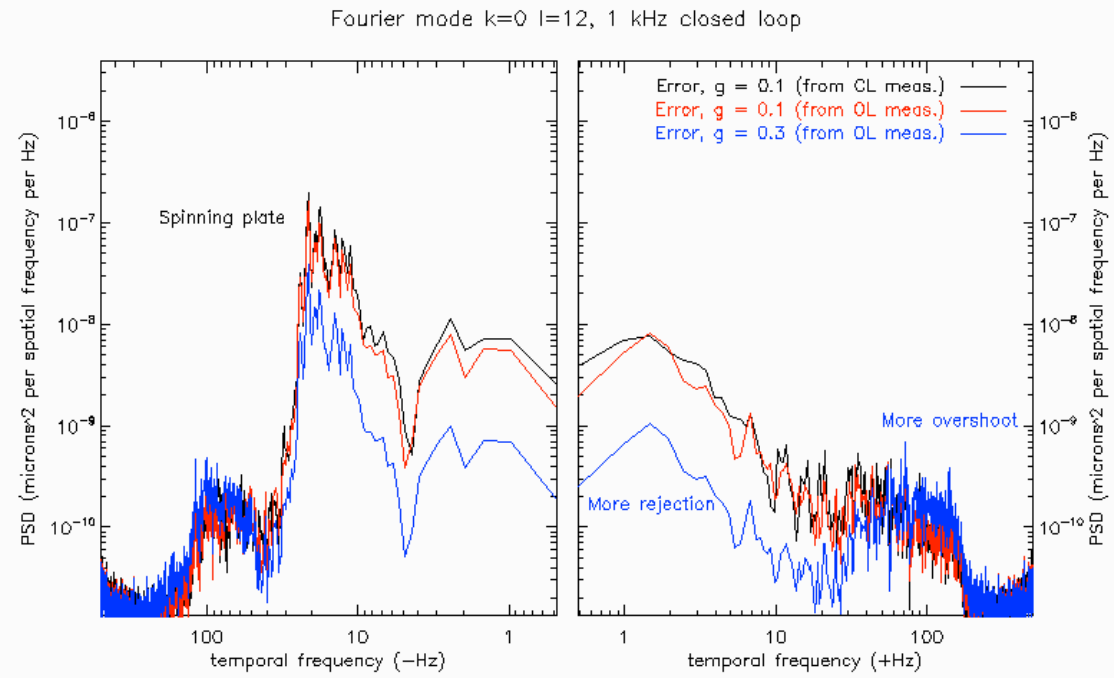

Figure 2. Error PSDs for same Fourier mode $k=0, l=12$. In black is the estimated error PSD from closed-loop operation (this is simply the measurement PSD from the previous figure, divided by the WFS response). In red is the estimate of the closed-loop error PSD from open-loop measurements. It agrees quite well with actual closed-loop behavior. In blue is the estimate for a higher gain of 0.3. Increasing the gain improves the low temporal frequency rejection and bandwidth but also increases the overshoot

performing as desired. Also in Figure 2 is structure from -20 to $-10 \mathrm{~Hz}$ that is due to the spinning phase plate. This is the error that a predictive controller could selectively correct; in our testbed due to the large size of the pupil and small size of the plate the frozen flow is more like 'frozen flow and rotation,' which smears out the peak more broadly then will be expected in actual operation.

This spatio-temporal frequency cube can be integrated to obtain the signal power (and RMS). Estimating performance at other gains from such a closed-loop measurement PSD cube is the essential function of the gain optimizer. An example of this is shown in Figure 2, where the blue curve is the estimate of the error PSD for a closed-loop gain of 0.3 .

To obtain predictions for performance at higher gains, we use the gain $=0.1$ closed-loop data set. First, the closed-loop PSD is converted to open-loop PSD using division by the systems Laplace model error transfer function. Then, since the SNR is so high, we assume this is actually the single signal open-loop PSD, not the joint signal + noise. We then apply the error transfer function with the new gain to get the temporal error component. The noise error component is estimated in the same way from the static phase plate trial data, using the noise transfer function with the new gain, of course. 


\subsection{Results}

First we analyze the measured residual phases in the spatial domain. Using noise only and signal-plus-noise tests, we can estimate the different components. As described above, we make corrections as necessary to go from measurement signal to error signal. Our results for conducted experiments are given in Table 1. In this case for $1 \mathrm{kHz}$ with a gain of 0.3 , we obtain a total error level of $46 \mathrm{~nm}$ RMS for the phase (no Tip-Tilt). For comparison, we also use the power-spectral method to estimate both actual tested performance and untested performance. Table 2 reflects our calculations of error RMS using the gains $=0.1$ closed-loop data. The protocol described above was used.

Note that the performance in the first row of Table 2 agree very closely with the temporal error in the first row of Table 1. This should happen, since the estimates are based on the closed-loop telemetry for that trial. The two other cases where we have a separate experiment in Table 1, the predictions in Table 2 (marked with *'s) are reasonable. Based on this we predict that with higher gains ( 0.4 for $1 \mathrm{kHz}, 0.3$ for $1.5 \mathrm{kHz}$, our temporal error would be in the between 34 and $38 \mathrm{~nm}$ RMS.

Once the AO system is integrated into the GPI instrument, these tests will be completed. There will be several changes in that system that we feel will improve the closed-loop performance. For our LLNL tests in August 2010, no spatial filter was used. This means that WFS measurements will have extra power due to aliasing that does not really exist. This means both our measured power and our estimates of performance are too high. Unfortunately, the exact discrepancy is not explicitly quantifiable.

The system was operated with an "engineering grade" Lincoln Labs CCDID-66 and SciMeasure camera electronics. These electronics had not yet been optimzied for the novel CCID-66 device. As a result, the $\mathrm{CCD} /$ cemra had substantial non-linearities, in particular variable subaperture slope gains that changed with flux and that were markedly different in the $\mathrm{x}$ - and $\mathrm{y}$-slopes. Furthermore, differences between amplifier regions injected significant bias, and even sometimes caused system instability due to actuator windup. Finally, system alignment will be improved; these LLNL tests were done with a residual 6 arcmin rotation between the WFS CCD pixels and the lenslet grid.

\begin{tabular}{l|c|c} 
& $1 \mathrm{kHz}$ & $1.5 \mathrm{kHz}$ \\
\hline Closed-loop, modal gains $=0.1$ & $\sigma_{t}=89, \sigma_{n}=9$ & $\sigma_{t}=76, \sigma_{n}=12$ \\
Closed-loop, modal gains $=0.2$ & not measured & $\sigma_{t}+\sigma_{n}=58$ \\
Closed-loop, modal gains $=0.3$ & $\sigma_{t}+\sigma_{n}=46$ & not measured \\
Closed-loop, modal gains $=0.4$ & not measured & not measured
\end{tabular}

Table 1. Residual dynamic error (nm RMS), temporal $\left(\sigma_{t}\right)$ and WFS noise $\left(\sigma_{n}\right)$ components, as measured during actual operation, strong phase plate spinning at setting 5000, WFS SNR $=3.3$.

\begin{tabular}{l|c|c} 
& $1 \mathrm{kHz}$ & $1.5 \mathrm{kHz}$ \\
\hline Closed-loop, modal gains $=0.1$ & $\sigma_{t}=89^{*}$ & $\sigma_{t}=78^{*}$ \\
Closed-loop, modal gains $=0.2$ & $\sigma_{t}=54$ & $\sigma_{t}=47^{*}$ \\
Closed-loop, modal gains $=0.3$ & $\sigma_{t}=40^{*}$ & $\sigma_{t}=38$ \\
Closed-loop, modal gains $=0.4$ & $\sigma_{t}=34$ & does not satisfy phase margin
\end{tabular}

Table 2. Estimated residual dynamic error (nm RMS), temporal and WFS noise components, using Laplace model of system and telemetry from closed-loop operation with modal gains $=0.1$. Strong phase plate spinning at 5000 , WFS $\mathrm{SNR}=3.3$. Boxes marked with ${ }^{*}$ directly compare to measured cases in the previous table. 


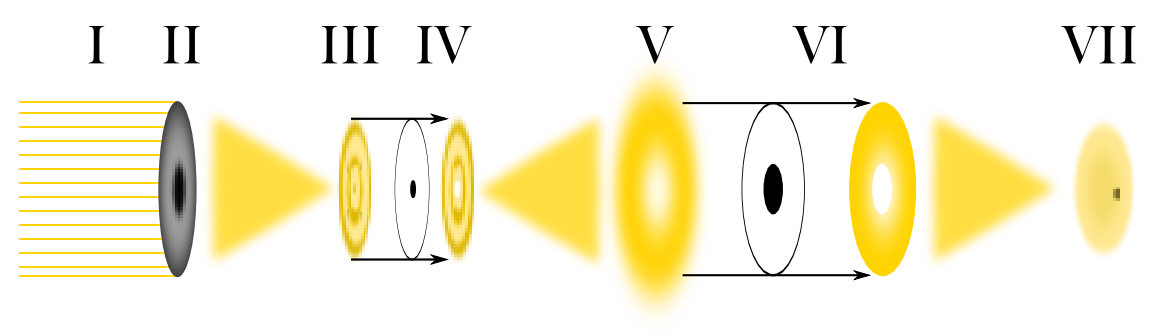

Figure 3. Schematic of the Apodized Pupil Lyot coronagraph. Light from the telescope and MEMS (I) enters the apodzier (II), with a donut-shaped transmission profile. This is focused into an Airy-like pattern (III). An opaque mask blocks the on-axis coherent beam (IV). In a re-imaged pupil (V), almost all the remaining light is diffracted outside the central aperture, and a slightly undersized Lyot stop blocks that (VI). In the final focal plane (VII) the starlight is mostly attenuated and an off-axis planet, which passes through all the stops, is visible.

\section{BAD ACTUATOR MITIGATION IN THE CORONAGRAPH}

Defective actuators on MEMS devices are a particular concern in a high-contrast coronagraphic adaptive optics system like GPI, since they will not just decrease the final Strehl ratio, but also scatter a persistent pattern of light into the "dark hole" in which we wish to detect extrasolar planets. The final point spread function (PSF) can be thought of as a combination of the diffraction pattern of the telescope and additional light scattered by errors phase and amplitude of the light propatating through this system. ${ }^{24}$ The coronagraph serves to block the diffracted portion of this. To first order, the final PSF is then approximated by the magnitude squared of the Fourier transform of the residual wavefront. A single stuck actuator with an approximately gaussian pupil will scatter light into a broad gaussian halo of angular width $N \lambda / D$ where $N$ is the number of actuators across the telescope pupil and $D$ is the pupil diameter. A pair of stuck actuators will produce a sine wave pattern; multiple stuck actuators will produce increasingly complex patterns of speckles that will completey swamp the signal of any planets.

Fortunately, for a small number of bad actuators, we can take advantage of the properties of the coronagraph to mitigate their effects. Figure 3 shows a schematic of the GPI coronagraph. It is an Apodized-Pupil Lyot Coronagraph (APLC) ${ }^{25}$ a modification of the original coronagraph devised by Lyot in 1929. The classic Lyot coronagraph uses an opaque mask in the center of the focal plane to block most of the coherent light from the onaxis star. In the next reimaged pupil plane, residual diffracted starlight is mostly concentrated at the edge of the pupil and can be blocked by a undersized pupil stop, called a Lyot stop. The APLC enhances this performance by pre-apodizing the pupil with a tapered transmission designed as an eigenfunction of the coronagraphic optical system, matched to the original pupil and focal stop; this has the effect of almost completely removing diffracted light within the original pupil, so the Lyot stop is not significantly undersized compared to the input.

There are at least three possible pupil planes for blocking a bad actuator: the MEMS itself, the entrance to the APLC at the apodization, or the Lyot plane. Blocking the bad actuator at the MEMS or apodizer pupil does not actually reduce scattered light, as the diffraction occurs at the edges of that block. The coronagraph relies on destructive interference from all the parts of the pupil to cancel the diffracted light - removing light from a bad actuator location means that light is no longer available for this interference, and results in as much or more light as would have been scattered by the actuator in the first place. Smoothly tapering the transmission profile around the bad actuator can redistribute this residual diffraction, but this merely serves to concentrate the light closer to the star, where planets would otherwise be found.

Instead, bad actuators can be effectively blocked in the final Lyot plane. One action of a coronagraph is to convert phase errors in the input pupil into intensity patterns in the output pupil; a defective actuator turns into a well-defined bright region in the final Lyot plane. This is illustrated in Figure 4. This can be crudely understood in the geometric optics limit - rays from the bad actuator will be deflected and not focus in the center of the focal plane, hence missing the focal plane mask, and then will be reimaged to their original location in the 


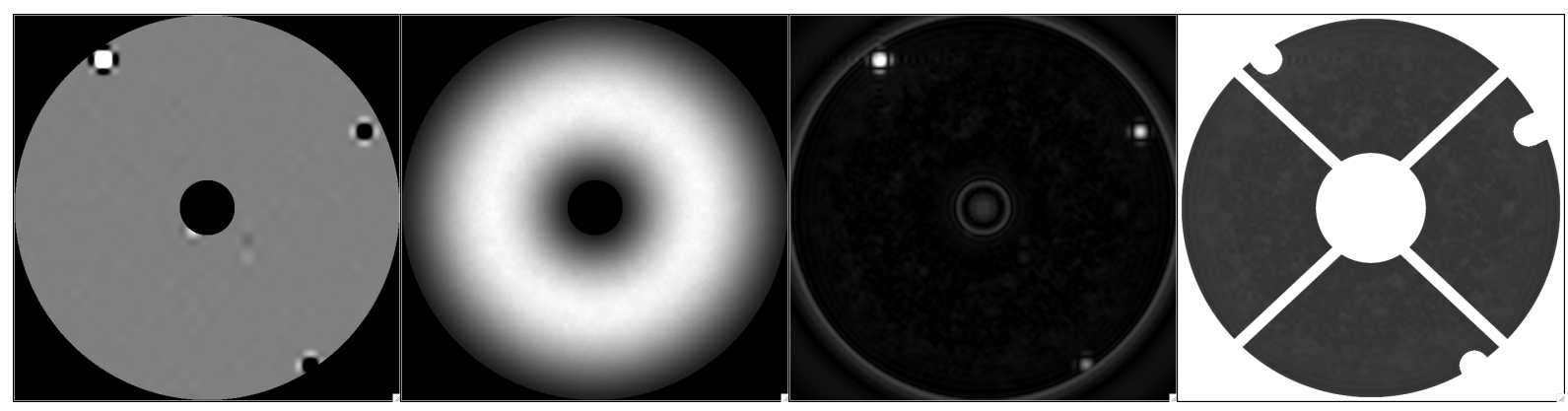

Figure 4. Propagation through the APLC. The first two panels show the wavefront phase and intensity as the beam enters the coronagraph, including the bad actuators on the MEMS and typical GPI internal optical errors. The third panel shows the intensity in the output Lyot pupil plane of the coronagraph, which has removed the coherent starlight but has bright patches remaining at the locations of the bad actuators. The final panel shows the Lyot plane after the custom Lyot mask (white).

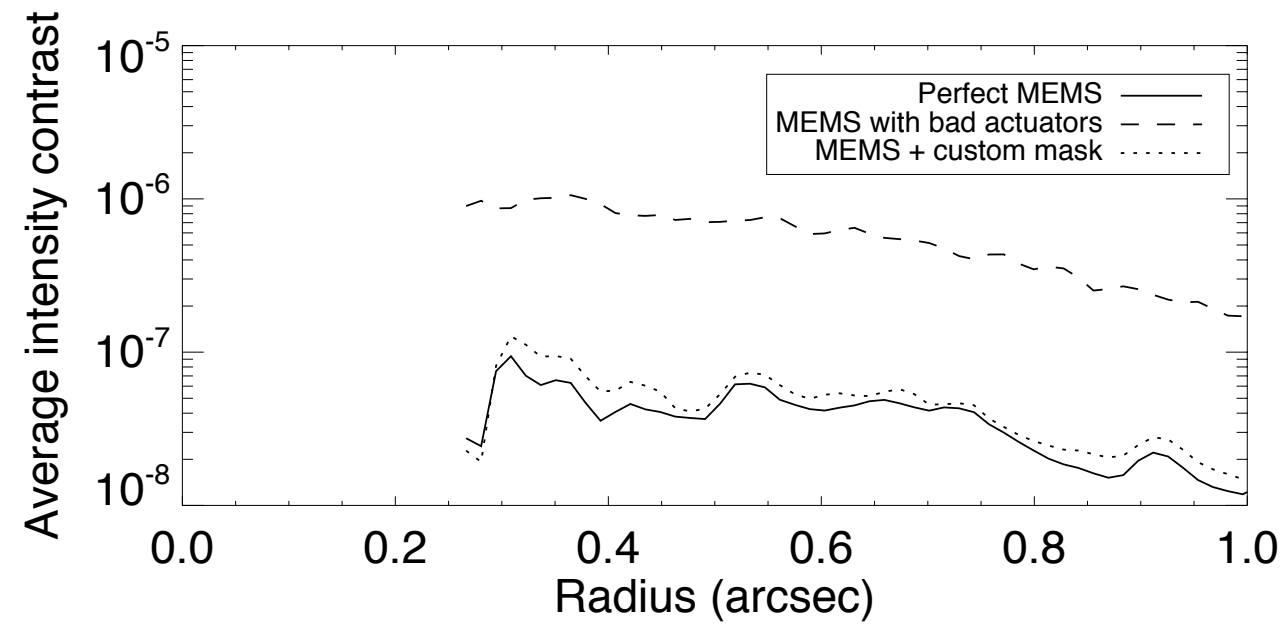

Figure 5. Radially-averaged contrasts from simulated science image PSFs, comparing a MEMS with no defects (solid) to a MEMS with defects as shown in Figure 4. Bad actuators (dashes) and special custom Lyot-plane masking (dots). Custom Lyot masking restores final science contrast to only 10 to $20 \%$ worse than no bad actuators.

final Lyot pupil. In Fraunhoffer optics the process is somewhat more complicated - the residual light is located in both a sharp image of the bad actuator and a broad smooth halo - but the net effect is the same. In this plane, a sharp-edged mask blocking the bad actuator is effective. The coronagraph has removed almost all of the coherent part of the starlight, so there is very little light present to diffract off the edges of the mask. To allow for residual diffraction and alignment errors, a typical size for these masks would be a radius of 1.5 actuators.

Manufacturing these masks presents a challenge. The GPI MEMS has a small enough number of defective actuators that they can be located near the edge of the pupil. As a result, the mask can be manufactured as a continuous piece. The GPI Lyot pupil has a size of $10 \mathrm{~mm}$, so the individual bad actuator blocks would have a diameter of 680 microns, easily within the capabilities of electron dischage machining. The mask must also be aligned to within a small fraction of an actuator spacing, but GPI has precisely actuated piezoelectric steering mirrors that can be used for this task.

Figure 5 shows a simulation of a coronagraph operating with the science-grade MEMS selected for GPI. Operation with unmasked abnormal actuators on this device results in a factor of 10 loss in contrast, which would be debilitating. Masking in the Lyot stop plane is quite effective - final contrast (detectable planet brightness) is reduced by only $10-20 \%$ from the no-defect DM case. 


\section{CONCLUSIONS}

The final GPI MEMS DM was produced by a multi-year research effort by BMC. The device has significantly more stroke and many more actuators than were possible at GPI's conceptual design five years ago. Manufacturing issues on large arrays remain a challenge; a device with no abnormal actuators in the 44-actuator across GPI pupil was not achieved.

Essential steps to using a MEMS DM in a high-performance AO system include using Woofer-Tweeter control to compensate for inadequate stroke, pre-correcting for the influence function of the DM, precisely calibrating the voltage-phase relationship of each actuator, dealing with abnormal actuators, and addressing Talbot effects in the $\mathrm{AO}$ and WFS relays.

An engineering-grade 4K MEMS was integrated with the GPI Woofer, an engineering-grade WFS CCD and the real-time control computer at LLNL in August 2010. Closed-loop tests with a spinning phase plate (approximately median seeing) and at a high SNR, demonstrated as little as $46 \mathrm{~nm}$ RMS phase error, for both temporal and noise error terms. Use of system telemetry confirms these numbers and provides an estimate of as low as $34 \mathrm{~nm}$ RMS for higher gains.

The few remaining abnormal actuators in GPI's science-grade MEMS pose a large threat to final science contrast if left uncorrected. We presented the principles of a technique which masks the abnormal actuator locations in the Lyot-stop plane. Simulations show that such masking can restore contrast to near no-defect levels.

The final science-grade GPI MEMS will be integrated with the science-grade WFS and the rest of the instrument at the UCSC Lab for Adaptive Optics beginning in January 2011.

\section{ACKNOWLEDGMENTS}

This work performed under the auspices of the U.S. Department of Energy by Lawrence Livermore National Laboratory under Contract DE-AC52-07NA27344. The document number is LLNL-CONF-464837. This work has been supported by the National Science Foundation Science and Technology Center for Adaptive Optics, managed by the University of California at Santa Cruz under cooperative agreement No. AST - 9876783. This work has been supported by the National Science Foundation under cooperative agreement No. AST - 0084699.

\section{REFERENCES}

[1] B. A. Macintosh, J. R. Graham, D. W. Palmer, R. Doyon, J. Dunn, D. T. Gavel, J. Larkin, B. Oppenheimer, L. Saddlemyer, A. Sivaramakrishnan, J. K. Wallace, B. Bauman, D. A. Erickson, C. Marois, L. A. Poyneer, and R. Soummer, "The gemini planet imager: from science to design to construction," Proc. SPIE 7015, p. $701518,2008$.

[2] L. A. Poyneer and B. Macintosh, "Spatially filtered wave-front sensor for high-order adaptive optics," $J$. Opt. Soc. Am. A 21, pp. 810-819, 2004.

[3] T. Fusco, C. Petit, G. Rousset, J.-F. Sauvage, K. Dohlen, D. Mouillet, J. Charton, P. Baudoz, M. Kasper, E. Fedrigo, P. Rabou, P. Feautrier, M. Downing, P. Gigan, J.-M. Conan, J.-L. Beuzit, N. Hubin, F. Wildi, and P. Puget, "Design of the extreme AO system for SPHERE, the planet finder instrument of the VLT," Proc. SPIE 6272, p. 62720K, 2006.

[4] B. Macintosh, J. Graham, L. Poyneer, G. Sommargren, J. Wilhelmsen, D. T. Gavel, S. Jones, P. Kalas, J. P. Lloyd, R. Makidon, S. S. Olivier, D. Palmer, J. Patience, M. Perrin, S. Severson., A. Sheinis, A. Sivaramakrishnan, M. Troy, and J. K. Wallace, "Extreme adaptive optics planet imager: XAOPI," Proc. SPIE 5170, pp. 272-282, 2003.

[5] J. W. Evans, K. Morzinski, L. Reza, S. Severson, L. Poyneer, B. Macintosh, D. Dillon, G. Sommargren, D. Palmer, D. Gavel, and S. Olivier, "Extreme adaptive optics testbed: High contrast measurements with a MEMS deformable mirror," Proc. SPIE 5905, p. 59050Y, 2005.

[6] J. W. Evans, K. Morzinski, S. Severson, L. Poyneer, B. Macintosh, D. Dillon, L. Reza, D. Gavel, D. Palmer, S. Olivier, and P. Bierden, "Extreme adaptive optics testbed: performance and characterization of a 1024MEMS deformable mirror," Proc. SPIE 6113, p. 61130I, 2006. 
[7] K. M. Morzinski, J. W. Evans, S. Severson, B. Macintosh, D. Dillon, D. Gavel, C. Max, and D. Palmer, "Characterizing the potential of MEMS deformable mirrors for astronomical adaptive optics," Proc. SPIE 6272, p. 627221, 2006.

[8] J. W. Evans, B. Macintosh, L. A. Poyneer, K. Morzinski, S. Severson, D. Dillon, D. Gavel, and L. Reza, "Demonstrating sub-nm closed loop MEMS flattening," Opt. Exp. 14, pp. 5558-5570, 2006.

[9] K. M. Morzinski, B. A. Macintosh, D. Dillon, D. Gavel, D. Palmer, and A. Norton, "Empirical measurement of MEMS stroke saturation, with implications for woofer-tweeter architectures," in Adaptive Optics Systems, N. Hubin, C. E. Max, and P. L. Wizinowich, eds., Proc. SPIE 7015, p. 70153N, SPIE, 2008.

[10] K. M. Morzinski, B. Macintosh, D. Gavel, and D. Dillon, "Stroke saturation on a mems deformable mirror for woofer-tweeter adaptive optics," Opt. Express 17, pp. 5829-5844, Mar 2009.

[11] A. Norton, J. W. Evans, D. Gavel, D. Dillon, D. Palmer, B. Macintosh, K. Morzinski, and S. Cornelissen, "Preliminary characterization of boston micromachines' 4096-actuator deformable mirror," in $M E M S$ Adaptive Optics III, S. S. Olivier, T. G. Bifano, and J. A. Kubby, eds., Proc. SPIE 7209, p. 72090I, SPIE, 2009.

[12] T. Bifano, "Personal communication." Boston University, 2010.

[13] J.-F. Lavinge and J.-P. Véran, "Woofer-tweeter control in an adaptive optics system using a Fourier reconstructor," J. Opt. Soc. Am. A 25(9), pp. 2271-2279, 2008.

[14] J. W. Hardy, Adaptive Optics for Astronomical Telescopes, Oxford University Press, New York, 1999.

[15] K. M. Morzinski, K. B. W. Harpsœ, D. T. Gavel, and S. M. Ammons, "The open-loop control of MEMS: modeling and experimental results," Proc. SPIE 6467, p. 64670G, 2007.

[16] K. M. Morzinski, D. T. Gavel, A. P. Norton, D. R. Dillon, and M. R. Reinig, "Characterizing MEMS deformable mirrors for open-loop operation: high-resolution measurements of thin-plate behavior," Proc. SPIE 6888, p. 68880S, 2008.

[17] C. R. Vogel and Q. Yang, "Modeling, simulation, and open-loop control of a continuous facesheet MEMS deformable mirror," J. Opt. Soc. Am. A 23, pp. 1074-1081, 2006.

[18] C. Vogel, G. Tyler, Y. Lu, T. Bifano, R. Conan, and C. Blain, "Modeling and parameter estimation for point-actuated continuous-facesheet deformable mirrors," J. Opt. Soc. Am. A 27(11), pp. A56-A63, 2010.

[19] J. B. Stewart, A. Diouf, Y. Zhou, and T. Bifano, "Open-loop control of a MEMS deformable mirror for large-amplitude wavefront control," J. Opt. Soc. Am. A 24, pp. 3827-3833, 2007.

[20] L. A. Poyneer, A. Norton, and D. Dillon, "Open-loop shaping of a 4k mems with fourier-domain precompensation," in Adaptive Optics: Methods, Analysis and Applications, Adaptive Optics: Methods, Analysis and Applications, p. JTuC3, 2009.

[21] GLAD Theory Manual, Applied Optics Reserach, 2009.

[22] L. A. Poyneer, D. Dillon, S. Thomas, and B. A. Macintosh, "Laboratory demonstration of accurate and efficient nanometer-level wavefront control for extreme adaptive optics," Appl. Opt. 47, pp. 1317-1326, 2008.

[23] L. A. Poyneer and J.-P. Véran, "Optimal modal Fourier transform wave-front control," J. Opt. Soc. Am. A 22, pp. 1515-1526, 2005.

[24] A. Sivaramakrishnan, J. P. Lloyd, P. Hodge, and B. A. Macintosh, "Speckle decorrelation and dynamic range in speckle noise-limited imaging," Ap. J. 581, pp. L59-L62, 2002.

[25] R. Soummer, "Apodized Pupil Lyot Coronagraphs for Arbitrary Telescope Apertures," Ap. J. 618, pp. L161-L164, 2005. 